\title{
Laser Based Three-Dimensional Measurement of Entire Foot Shape During Motion
}

\author{
Matija JEZERŠEK ${ }^{* a}$, Janez MOŽINA ${ }^{a}$ \\ ${ }^{a}$ University of Ljubljana, Faculty of Mechanical Engineering;
}

\begin{abstract}
Method for measuring 3D foot shape in motion is presented. The entire foot is simultaneously measured from multiple viewpoints by four measuring modules. Measuring principle is based on multiple-laser-line triangulation, where the object is illuminated with grating-like laser light pattern. Each module uses unique laser wavelength which prevents unwanted overlapping effects between adjacent light patterens. The precision of the system is $0.3 \mathrm{~mm}$. It is capable of measuring objects in motion, which is demonstrated in the paper.
\end{abstract}

Keywords: foot-shape measurement, laser triangulation, 3D high speed measurement, multiple-line illumination.

\section{Introduction}

Optical three-dimensional shape measurement of live objects is becoming an important developing and research tool because of its non-intrusive nature and high measuring speed. The current methods are reaching truly high speed in one view configuration, but in case of the entire object shape measurement, they are limited due to mutual interference between multiple measuring modules. Kimura et al. [1] demonstrates a method for 3D foot shape measurement deformation in motion, which is based on a projector-camera system with color-coded fringe projection. Although they build a system with multiple-view configuration, the foot can be measured only from one direction at the same time. By using one view measuring setup, there is always the shading problem inherent in the one-view based techniques [2].

The presented method overcomes this limitation by using a laser multiple-line triangulation technique, where each of four measuring modules is using a unique laser wavelength and narrow-band filters placed on cameras [3]. In this manner, unwanted overlapping between adjacent light patterns is solved and consequently the measuring speed is limited only by the frame rate of the selected cameras.

\section{Measuring set-up}

The measuring modules consist of a laser projector and a digital camera (see Fig. 1). The laser projector generates an illumination pattern from 33 equally separated light planes that are directed at the surface. The camera records the illuminated surface from a different viewpoint, and the light pattern is distorted by the shape of the surface. An interference filter (10 nm FWHM) is placed between the lens and the camera's CCD sensor to transmit only the laser light of the corresponding projector. 


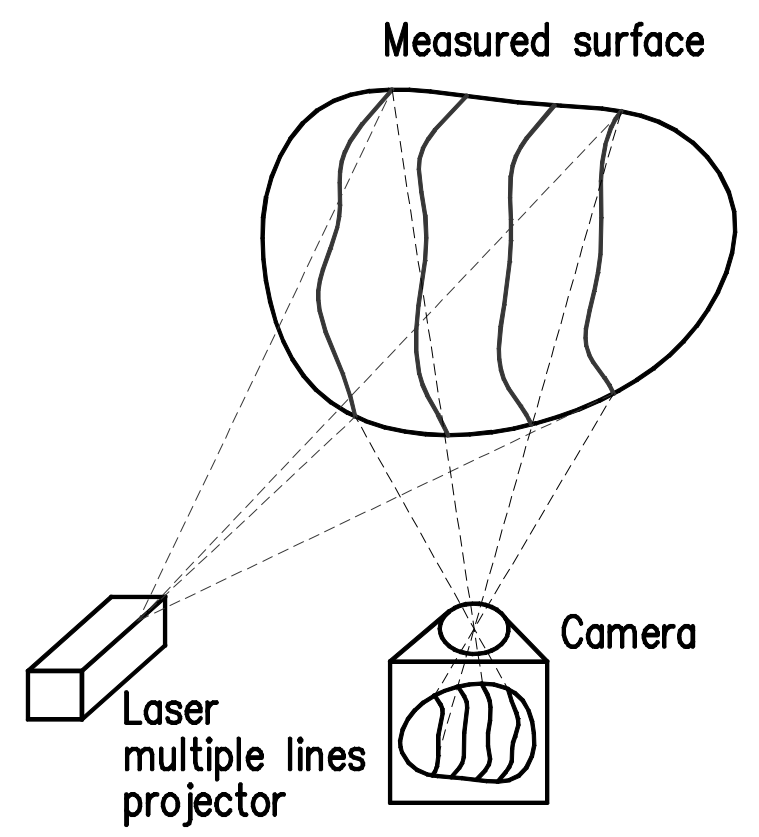

Fig. 1. Laser multiple-line triangulation principle.

The measuring system is an assembly of four measuring modules, three mirrors and a connecting frame. The mirrors are used in the measuring system in order to minimize its overall dimensions. The measuring modules $(\mathrm{Mm1}, \ldots, \mathrm{Mm} 4)$ and their associated mirrors (Mi1, Mi2 and Mi3) are fixed to the casing frame in such a way that the entire surface of the foot is in the field of view of at least one measuring module (see figure 7). The first mirror (Mi1) is positioned under the plane of the transparent supporting plate so that it is inclined with respect to the plane of the supporting plate. The remaining mirrors (Mi2 and Mi3) are symmetrically arranged in vertical planes with respect to the symmetrical plane of the system. They are placed near the entry aperture of the casing with a separation of $180 \mathrm{~mm}$ so that the foot can be easily inserted.

All modules have a triangulation angle $\wedge=20^{\circ}$, which means angular separation between camera and projector. The distance between the camera and the projector is $\sim 300 \mathrm{~mm}, 230 \mathrm{~mm}, 280 \mathrm{~mm}$ and 280 $\mathrm{mm}$ for Mm1, Mm2, Mm3, and Mm4, respectively. The lens focal length is $16 \mathrm{~mm}$, and the CCD dimension is $6.47 \times 4.83 \mathrm{~mm}$ with resolution of $640 \times 480$ pix. The laser projectors generate 33 light planes, which are equally separated for $\square \wedge=0.38^{\circ}$ and its fan angle is $30^{\circ}$.
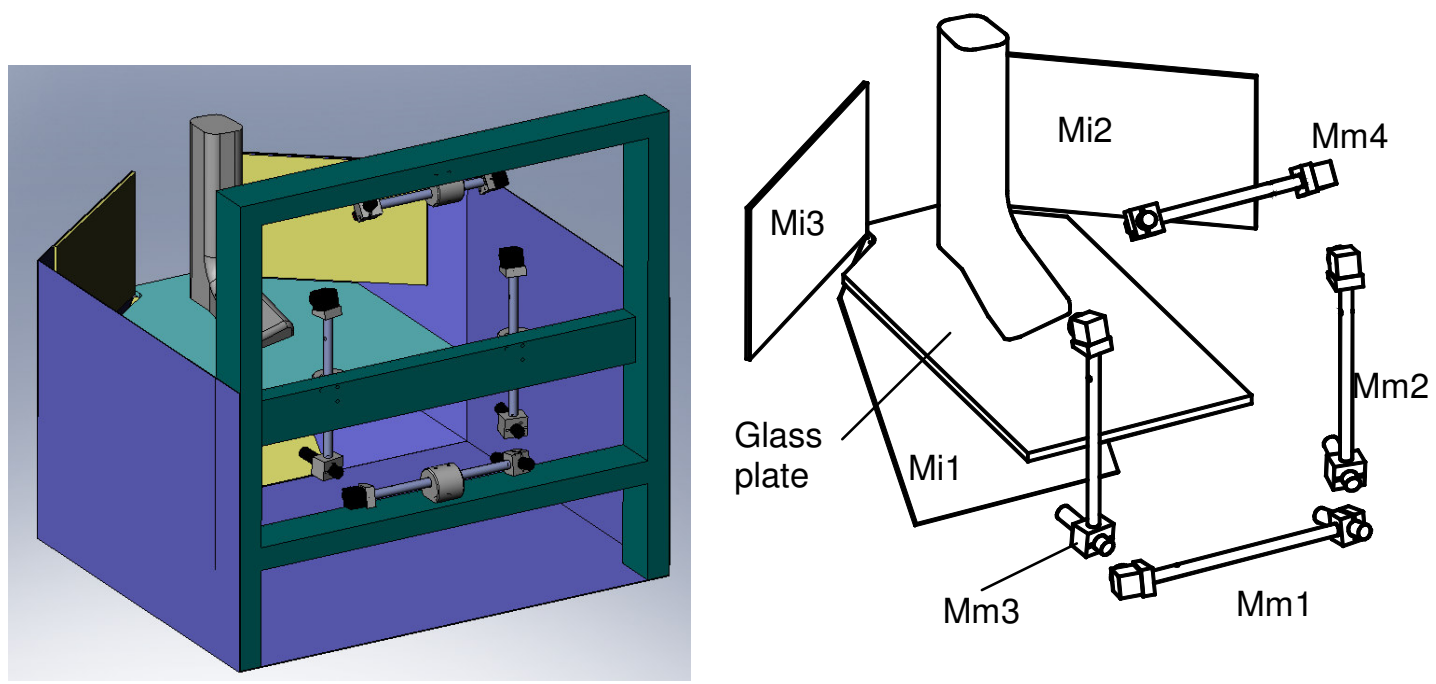

Fig. 2. Optomechanical part of foot-measuring system 
All four measuring modules can acquire object shape simultaneously by using the so-called colour modulation, where the laser projector and interference filter of each module are synchronized at unique wavelengths. In our case, the selected wavelengths are $635 \mathrm{~nm}, 650 \mathrm{~nm}, 670 \mathrm{~nm}$, and $685 \mathrm{~nm}$. Using this technique, all cameras can acquire the image at the same moment and there is no cross talking between neighbor fringe patterns. Digitized images of the illuminated foot are shown in Fig. 3. The acquisition time of the entire system equals to the shutter time of the camera, which is less than 20 msec.
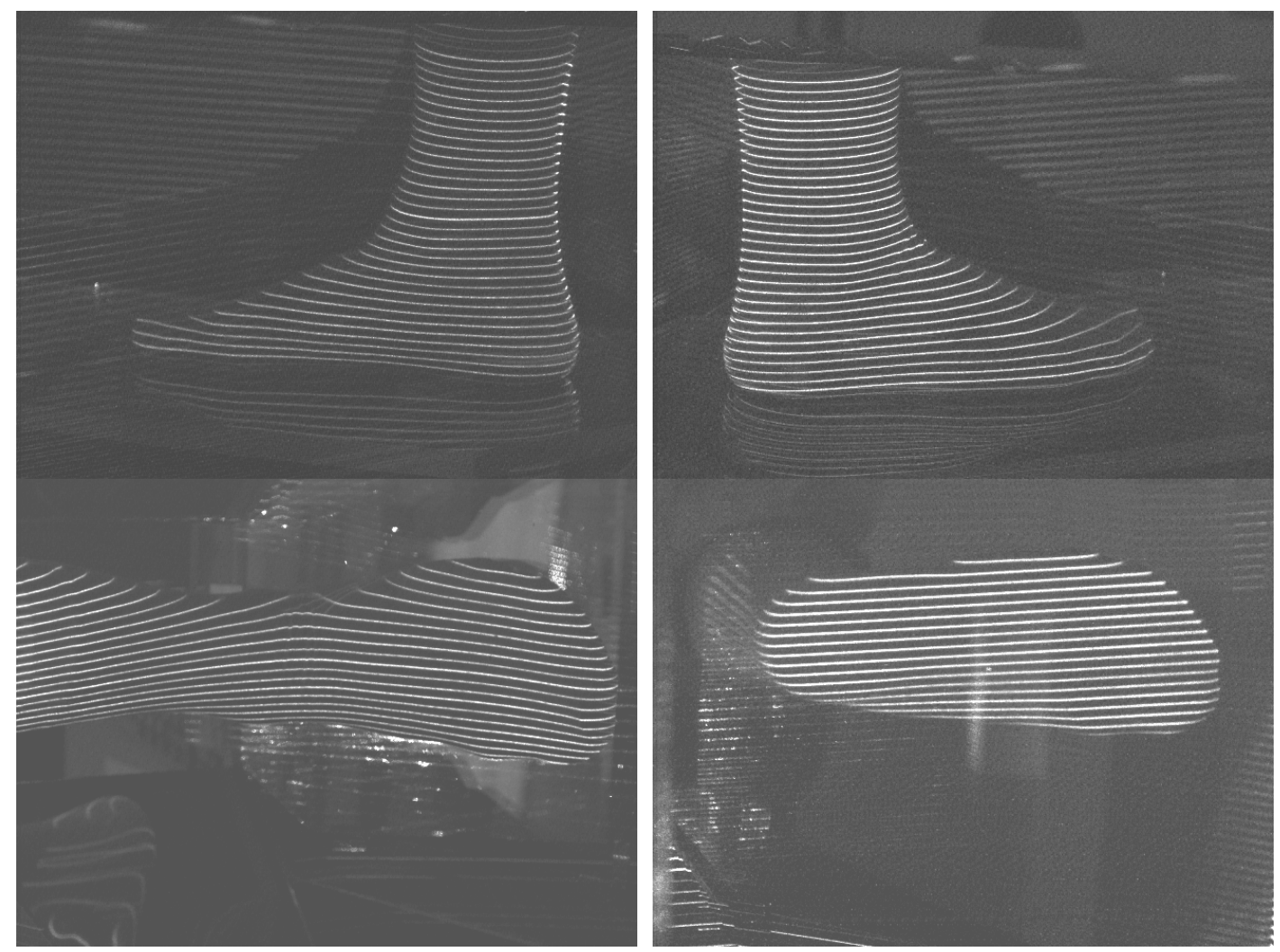

Figure 3: Images of illuminated foot acquired with cameras of each measuring module.

After the acquisition, the images are transferred to a personal computer, where the laser line segments in each image are detected via sub-pixel line-detection algorithm, which is based on the first-derivative zero-crossing of the pixel values perpendicular to the lines. The detected contours are afterwards sorted and indexed. After the line indexing, the transformation from two- to three-dimensional space is performed on every point of the detected line segment.
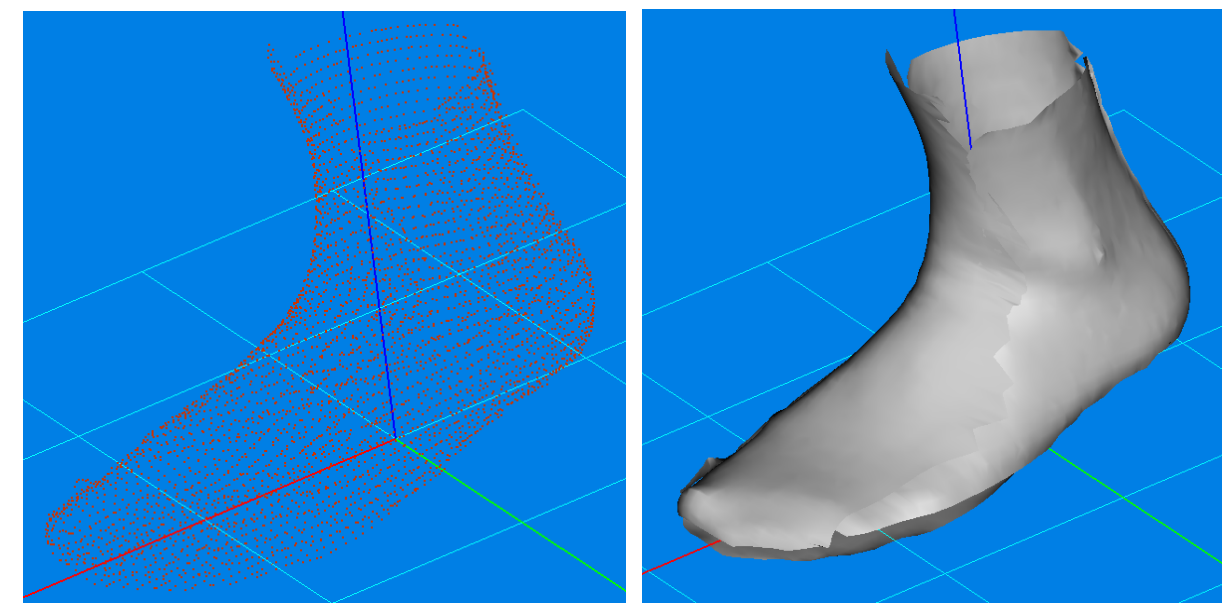

Figure 4: Measured foot. Left: point cloud; Right: shaded surface. 
Finally, the points in 3D space are triangulated, displayed (see Fig. 4), and analyzed with the intention to determine the various dimensions of the foot, such as the length, width, height, and perimeter for a particular section through the foot. The precision of the system is better than $\pm 0.3 \mathrm{~mm}$, which was determined during calibration process, where reference body was measured.

\section{Experiments}

Measurements of foot in motion were executed during performing typical human movement, such as step forward, jump, stepping up on toes etc. Figure 5 shows measurement of a foot while the person is stepping on their toes. The measurement repetition rate is $25 \mathrm{~Hz}$.
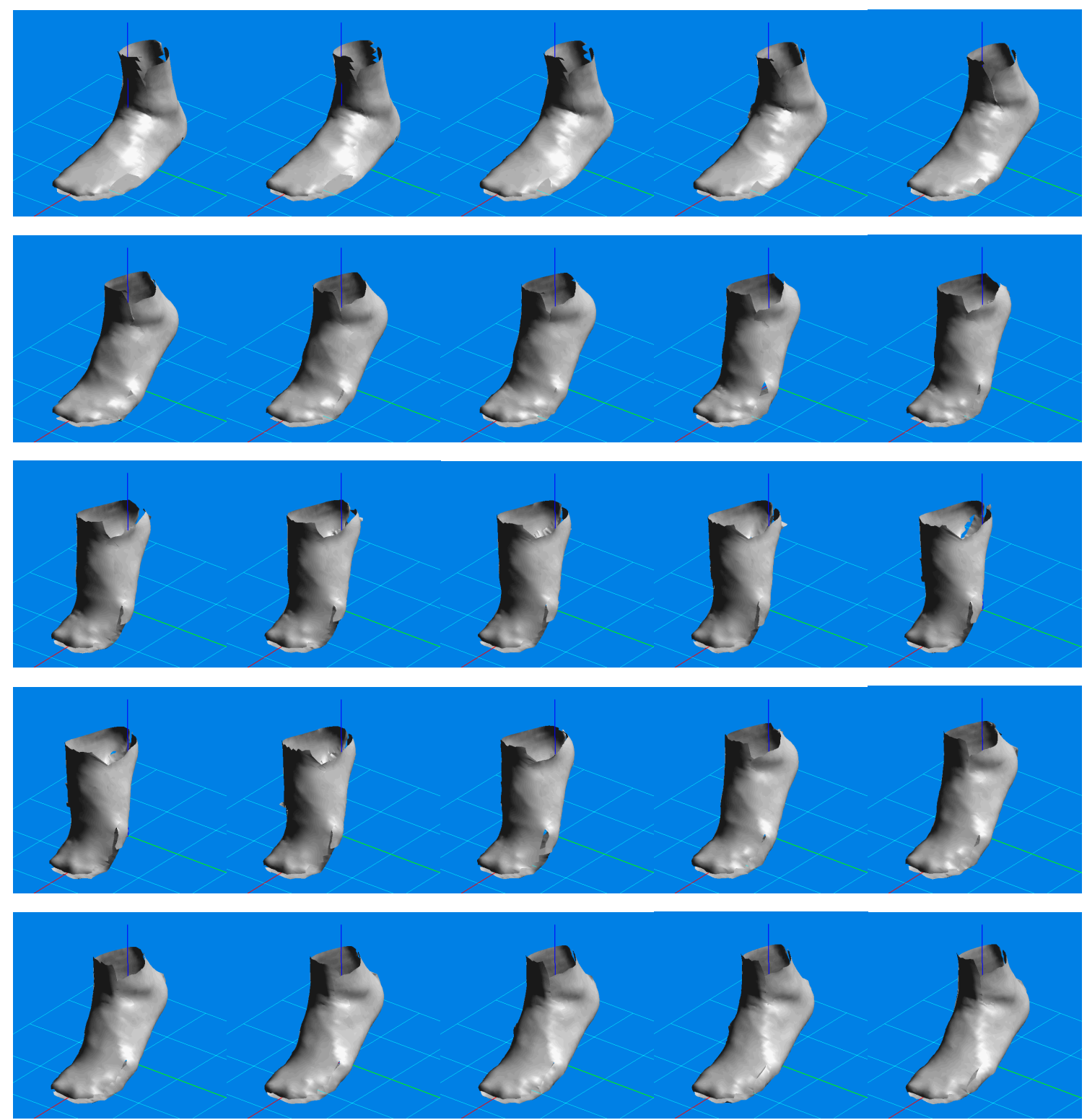

Figure 5: Three-dimensional foot shape acquired while the person is stepping up on their toes.

The next figure (Fig. 6) represents the sequence of the foot deformation while the person is jumping with both legs. It is evidently from both figures that the system is capable of measuring fast foot movement. The surface is well covered from all directions and details such as sock folds are visible. The current system has relatively narrow measurement range which is only $200 \mathrm{~mm}$ high, thus the foot have cropped edges on the upper side during high position. 

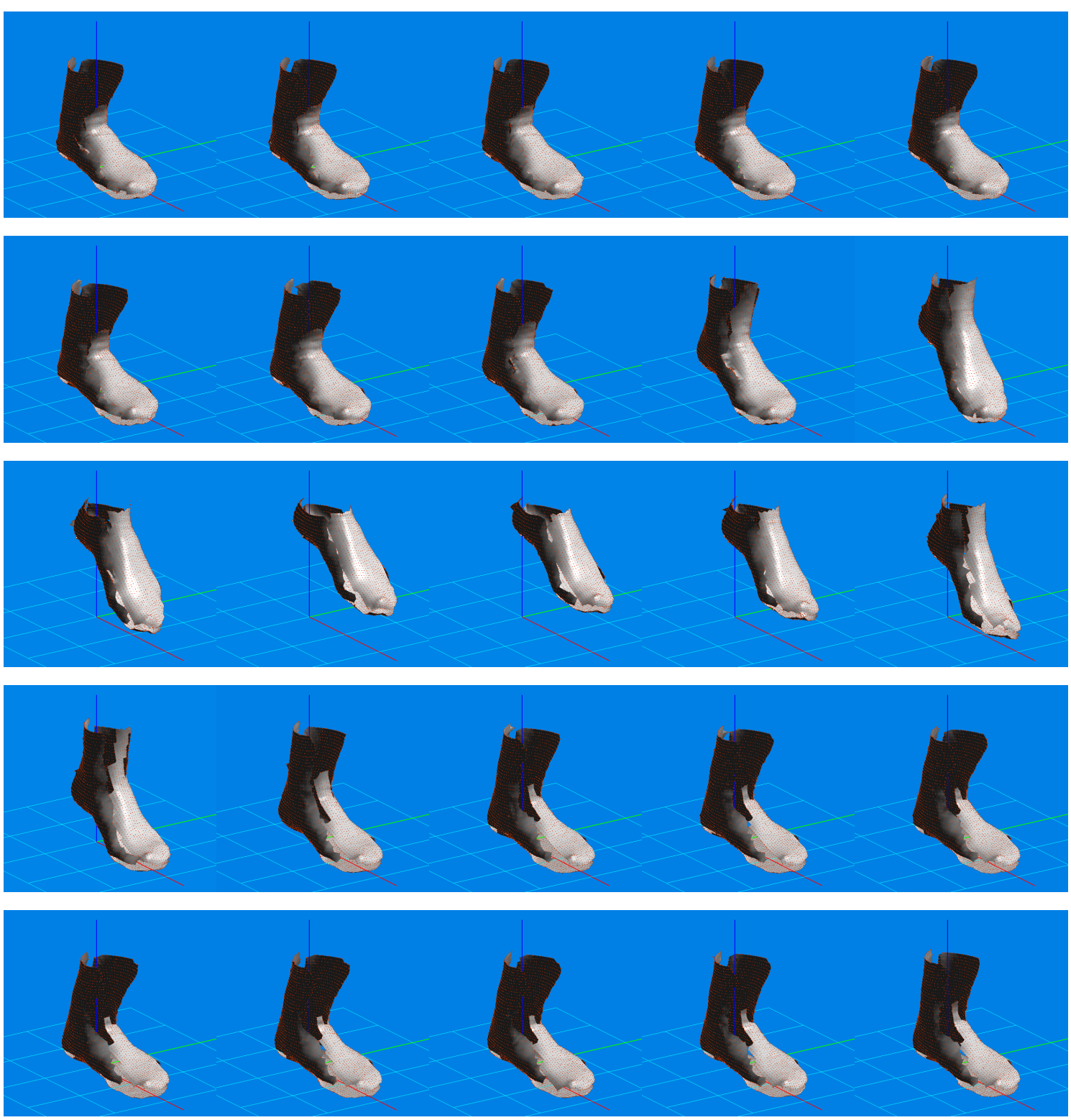

Figure 6: Three-dimensional foot shape acquired while the person is jumping with both legs.

\section{Conclusion}

The main advantage of the presented technique is the capability of measuring the foot from several viewpoints simultaneously, which enables dynamic 3D shape measurement. The measuring precision is $0.3 \mathrm{~mm}$ and the repetition rate is $25 \mathrm{~Hz}$, which is mostly determined by the camera frame rate. The new method is applicable not only in the footwear industry, but also in sport science, kinesiology, biomechanics and medical imaging where dynamic shape of the complete foot or any other body part is important. Further development is directed into enlargement of the measuring range and building a catwalk, which will enable us to measure foot shape also during walking and running.

\section{References}

1. M. Kimura, M. Mochimaru, T. Kanade, Measurement of 3D Foot Shape Deformation in Motion, Proceedings of the 5th ACM/IEEE International Workshop on Projector camera systems 2008, Marina del Rey, California, 2008.

2. Su XY, Zhang QC, Dynamic 3-D shape measurement method - A review, Optics and Lasers in Engineering 48 (2) 191-204 (2010).

3. Jezeršek M, Možina J, High-speed measurement of foot shape based on multiple-laser-plane triangulation, Opt. eng. 48(11) 113604-1-113604-8 (2009). 